\title{
Feasibility of different thermal analysis of FRP - reinforced concrete
}

\author{
by P. Corvaglia*; U. Galietti ${ }^{* *}$; A. Largo*; S. Nenna; L. Spagnolo* \\ "Department of Materials and Structures Engineering - CETMA Consortium, Brindisi, \\ Italy \\ ${ }^{\star \star}$ Dipartimento Ingegneria Meccanica e Gestionale - Politecnico di Bari, Bari, Italy
}

\begin{abstract}
The aim of this work is to develop a reliable and confident non-destructive testing technique by means on infrared thermography. In particular, pulse heating thermography and lock-in thermography as a tool for the evaluation of hidden defect in FRP-reinforced concrete structures were examined.

The study on a concrete sample strengthened with externally bonded FRP (Fiber Reinforced Plastic) sheets, with known defects appropriately applied at the FRP-concrete interface, is presented.
\end{abstract}

The results obtained with both procedures are compared.

\section{Introduction}

Externally bonded Fiber Reinforced Plastic (FRP) materials, thanks to their mechanical and physical properties (excellent strength-to-weight and stiffness-toweight ratios, high durability, corrosion resistance) are widely used for repairing, retrofitting and strengthening of existing structures in recent years. In fact, there may be several reasons for the need of strengthening and upgrading civil structures, such as expired design life, changes in functionality, damage caused by mechanical actions and environmental effects, more stringent design requirements, original design and construction errors.

However, the success of FRP reinforcement techniques is subjected to the perfect adhesion between the FRP and the underlying material. For this reason, it is essential to use Non-Destructive Testing (NDT) to asses the bond quality.

Among the NDTs, Infra-Red Thermography (IRT) is being given more and more attention, allowing the analysis of the effects induced by anomalies (i.e. voids or delaminations) on the thermal behaviour of the material under testing $[1,2,3,4,5]$.

The aim of this work is to develop a reliable and confident non destructive testing technique by means on infrared thermography for the evaluation of hidden defect in FRP-reinforced concrete structures; the presented study follows a previous stage of the authors' research [6], focused on the FRP-reinforced concrete structure analysis with thermocamera after an "impulsive heating".

The obtained results suggested to investigate on a different thermographic method, aiming at the detection of different kinds of defects, even those that pulsed thermography (PT) was not able to detect (i.e. delaminations).

In this paper a concrete sample strengthened by externally bonded FRP, with known defects appropriately applied at the interface, analysed with PT in [6], was analysed with lock-in thermography (LT).

The procedure requires a detailed set-up, in order to assess its feasibility. 


\section{Experimental set up}

According to the "ICC Evaluation Service" standards, a certain amount of defectiveness at the FRP-concrete interface can be accepted. In particular, air void on the order of $3.2 \mathrm{~mm}$ diameter and delaminations of less than $13 \mathrm{~cm}^{2}$, with a maximum of 10 delaminations per $1 \mathrm{~m}^{2}$, are allowed $[7,8]$. On the basis of such indications the CFRP-reinforced concrete sample was accurately prepared [6], imposing two types of defects with different shapes and dimensions (Fig. 1):
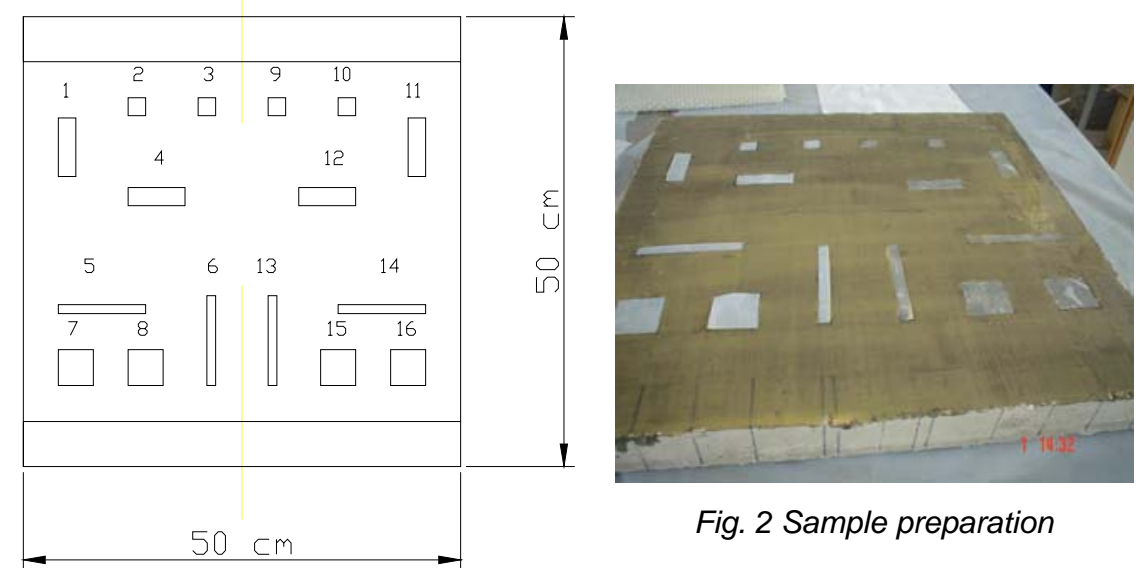

Fig. 2 Sample preparation

Fig. 1 Imposed known defects

- delaminations, by means of two bonded Teflon foils:

- defects $\mathrm{n}^{\circ} 5$ and $\mathrm{n}^{\circ} 6(100 \mathrm{~mm} \times 10 \mathrm{~mm})$;

- defects $\mathrm{n}^{\circ} 1$ and $\mathrm{n}^{\circ} 4(65 \mathrm{~mm} \times 20 \mathrm{~mm})$;

- defects $\mathrm{n}^{\circ} 7$ and $\mathrm{n}^{\circ} 8(40 \mathrm{~mm} \times 40 \mathrm{~mm})$;

- defects $\mathrm{n}^{\circ} 2$ and $\mathrm{n}^{\circ} 3(20 \mathrm{~mm} \times 20 \mathrm{~mm})$;

- lack-of-bonding of the FRP, by means of a simple Teflon foil:

- defects $\mathrm{n}^{\circ} 13$ and $14(100 \mathrm{~mm} \times 10 \mathrm{~mm})$;

- defects $\mathrm{n}^{\circ} 11$ and $12(65 \mathrm{~mm} \times 20 \mathrm{~mm})$;

- defects $\mathrm{n}^{\circ} 15$ and $16(40 \mathrm{~mm} \times 40 \mathrm{~mm})$;

- defects $\mathrm{n}^{\circ} 9$ and $10(20 \mathrm{~mm} \times 20 \mathrm{~mm})$.

The sample surface was analysed with to different methods: pulsed thermography (PT) [6] and lock-in thermography (LT).

In PT a thermal pulse is applied to the material to be inspected; by measuring the temporal evolution of temperature on the specimen surface with an infrared camera, subsurface defects can be revealed. A commercial halogen lamp with a nominal power of $500 \mathrm{~W}$ was used; the adopted thermocamera was a commercial microbolometric FLIR System with a 320x256 Focal Plane Array $[2,6]$.

LT uses thermal waves, instead of pulse, propagating inside the sample under testing. These waves will be reflected on parts where the physical heat propagation parameters are changing (i.e. at voids or delaminations). The interference pattern 
generated on the object surface by incoming and reflected waves is detected by an infrared camera; defective areas will exhibit a different phase with respect to sound areas [2]. A commercial halogen lamp with a nominal power of $1000 \mathrm{~W}$ was used, with a Deltatherm DT 1560 thermocamera.

The experimental setups were meant to be as cheap as possible, in order to develop an experimental procedure that could be easily applied on-the-field and not only in research laboratories.

\section{Pulsed thermography analytical results}

Thermographic data were processed with Almond's half maximum contrast method as described in [6]; a typical thermogram of the CFRP-reinforced sample is presented in Fig. 3. Two important set up parameters were investigated:

- the "impulsive heating" time, named Heating Time (HT);

- the time, after the heating step, at which the thermogram should be recorded, named Observation Time (TObs).

Partial results are reported in Fig. 4.

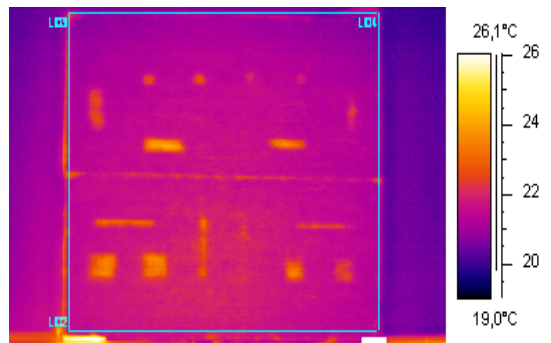

Fig. 3 Thermogram of the CFRP sample with imposed defects

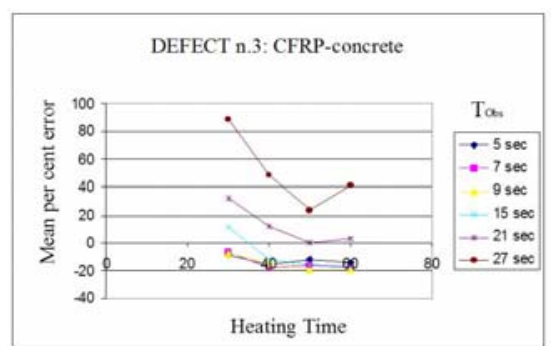

Fig. 4 Percentage error in the assessment of defect $n .3$ for different experimental set-up

Some interesting remarks can be pointed out:

- the error, defined as the difference between the estimated and the real number of pixels relative to a defective area, divided by the real number of pixels relative to the same defective area, increases with increasing TObs;

- Statistical ANalysis Of VAriance (ANOVA) indicates that the investigated parameters (TObs and HT), have no mutual influence;

- thermograms recorded at a TObs of about 20 s are not useful;

- the lower percentage error can be obtained with a HT period of 40-60 s.

\subsection{Neural Networks for defects evaluation}

In order to develop an automated procedure to be used by unskilled operator, a Neural Networks (NN) based method for data post-processing was implemented [6].

A careful investigation of different possible NN was carried out. The common elements of the investigated $\mathrm{NN}$ architecture were: 
- the use of three neuron layers (input layer, middle layer and output layer);

- the "logsig" transfer functions;

- the number of neurons at the last layer, equal to the number of pixel of the region under investigation.

Three different NN (named "CanetXX" where XX is the number of neurons in the input and middle layers) were analysed. All the NN were trained with the same set of data.

The "Canet70" NN was able to evaluate most analysed defects with a remarkable accuracy (see Fig. 5 as an example).

The feasibility of using Canet70 also for FRP-reinforced concrete samples different from those used for training was evaluated. Nevertheless, as visible in Fig. 6 , such feasibility is not guaranteed.

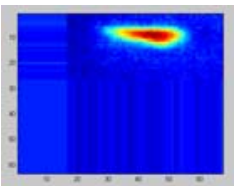

a): Th.Img defect $n^{\circ} 4$ Output of canet $70: 0,00 \%$ error

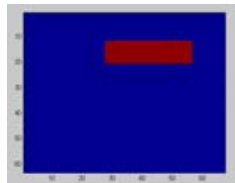

NN output

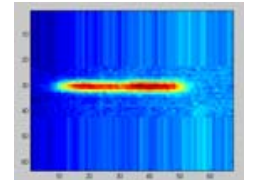

a): Th.Img defect $n^{\circ} 5$

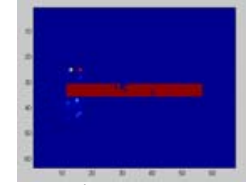

b) NN output

Fig. 5 Canet 70 results for CFRP-reinforced concrete sample

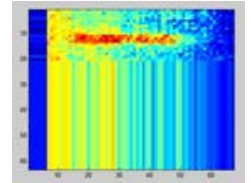

a): Th.Img defect $n^{\circ} 14$

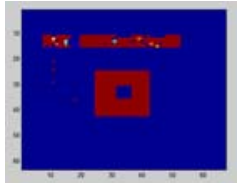

b) NN out put

Output of canet 70

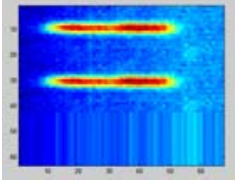

a): Th.Img two defects

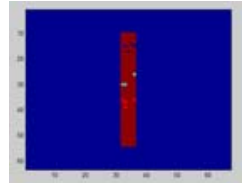

b) NN out put

Fig. $6 \mathrm{NN}$ analysis of GFRP-reinforced concrete sample (left) and two-defect in the same investigated region (right)

\section{$4 \quad$ Lock-in thermography analysis}

\subsection{Basic principles}

Lock-in thermography (LT) is based on thermal waves generated inside a specimen and remotely detected. Wave generation, for example, is performed by periodic heat deposition on a specimen surface (e.g., through sine-modulated lamp heating) while the resulting oscillating temperature field in the stationary regime is remotely recorded trough thermal infrared emission.

If the wave reaches areas of the object where the thermophysical properties change (e.g. at delaminations or inclusions), it is partly reflected, thus interfering with the incoming wave and causing an interference pattern in the local surface temperature.

By evaluating the amplitude and the phase of the local surface temperatures, information about the internal structure of the specimen under testing are allowed. 
In particular, lock-in refers to the necessity to exactly monitor the time dependence between the output signal and the reference input signal (i.e. the modulated heating).

Phase images are related to the propagation time, and since they are relatively insensitive to local optical surface features (such as non-uniform heating), they are interesting for NDT purposes.

The depth range of images is inversely proportional to the modulation frequency, so that higher modulation frequencies restrict the analysis in a nearsurface region [2].

A schematic illustration of the lock-in thermographic system used is shown in Fig. 7; sinusoidal reference signal for the lock-in measurement was generated in the computer and transferred to the lock-in heater control unit, which controlled a $1000 \mathrm{~W}$ halogen lamp via heater control relay.

According to Fig. 7 both the IR scanner and the lamp are located on the same side with respect to the specimen surface.

Periodical heating was conducted by alternatively switching the lamp heaters, according to the reference signal. Infrared radiation data from the specimen surface were measured by an infrared camera (DeltaTherm DT 1560), and transferred to the computer for the analysis.

Changes in phase and amplitude of the surface interference pattern will enable defect characteristics to be determined.

As it is known that the amplitude is insensitive to environmental reflections, while the phase is insensitive also to local power density or thermal emission coefficient, in the presented paper the attention is focused on phase images only.

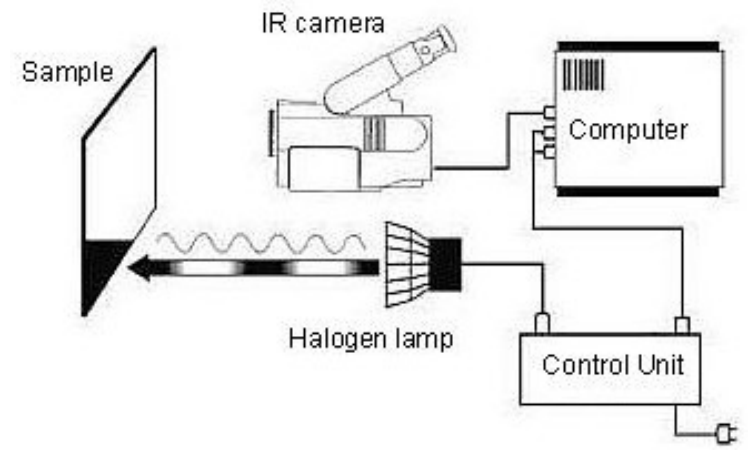

Fig. 7 Schematic illustration of $L T$ system

\subsection{Statistical analysis of variance (ANOVA)}

The defect visibility depends on several parameters; in the presented study several tests were carried out in order to find out the optimal parameters combination that allows the best defect visibility.

The influence of three parameters were investigated:

- heating time, with three possible levels (20 s, 30 s e 40 s),

- start frame, with three possible levels (1, corresponding to heating start, 1.25 , corresponding to a $1 / 4$ period shifting after heating start, and 1.5 , corresponding to a $1 / 2$ period shifting after heating start),

- number of sampled cycles, with three possible levels (4, 4.5 and 4.75), 
also considering the defect type (lack-of-bonding, A, or, delamination B), the phase image (with or without smoothing) and the dependent variable chosen among the following:

- contrast $=\bar{\phi}_{\text {def }}-\bar{\phi}_{\text {sou }}$

where:

$\bar{\phi}_{\text {def }}$ and $\bar{\phi}_{\text {sou }}$ are the phase mean value referred to a sound and a defective lines respectively (Fig. 8);

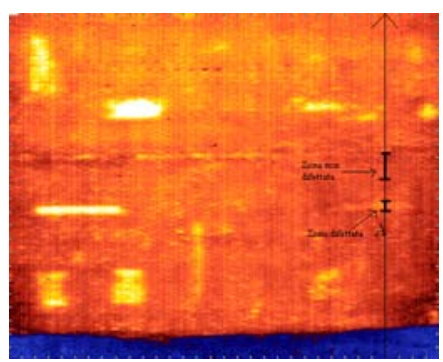

Fig. 8 Sound and defective areas for $A$ defect

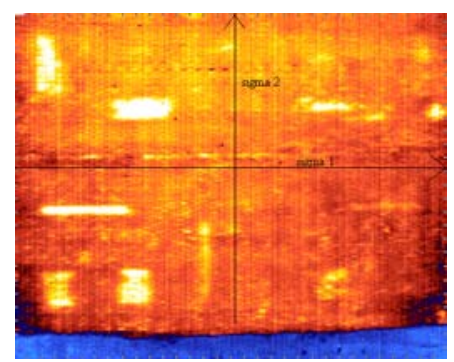

Fig. 9 Reference line for the normalized contrast definition

- $\operatorname{norm}$ contrast $=\frac{\bar{\phi}_{d e f}-\bar{\phi}_{\text {sou }}}{\bar{\sigma}_{n d}}$

where:

$\bar{\sigma}_{n d}=\frac{\sigma_{1}+\sigma_{2}}{2}$ is the average standard deviation of $\sigma_{1}$ and $\sigma_{2}$ referred to two lines on the phase image (Fig. 9); it gives a measure of the recorded image noise, as the hidden defects become more visible with decreasing the noise;

- $\max$ contrast $=\bar{\phi}_{\text {def } \max }-\bar{\phi}_{\text {sou }}$

where:

$\bar{\phi}_{d e f_{\max }}$ is the maximum phase value referred to a line crossing the defect;

- $\max$ norm contrast $=\frac{\bar{\phi}_{d e f_{\max }}-\bar{\phi}_{\text {sou }}}{\bar{\sigma}_{n d}}$.

The obtained results can be summarized as follows (Fig. 10 and Fig. 11):

- the normalized contrast and the maximum normalized contrast get their maximum value for $(n+0.75)$ sampled cycles and the start frame at level 1 ;

- the results obtained for A and B defect type are in good agreement.

Several works concerning $\mathrm{LT}$ are presented in literature and the authors always suggest to use a whole number of sampled cycles to enhance defect visibility. 


\section{http://dx.doi.org/10.21611/qirt.2006.098}

However, thanks to the results obtained from the presented experimental tests, it can be pointed out that, with a sampled cycles number equal to $(n+0.75)$ and with the start frame in correspondence to heating start, the images are characterized by a lower contrast value, but, at the same time, also by a lower noise value. This allows the defect to be more visible (see Fig. 12 as an example).
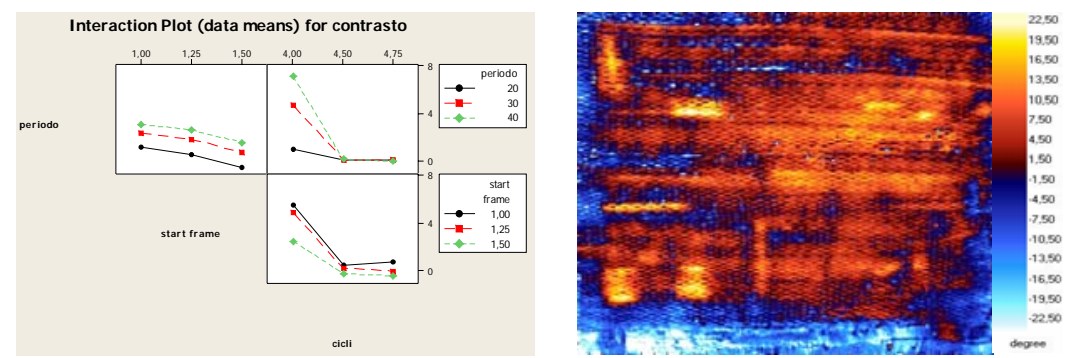

Fig. 10 Contrast interaction plot
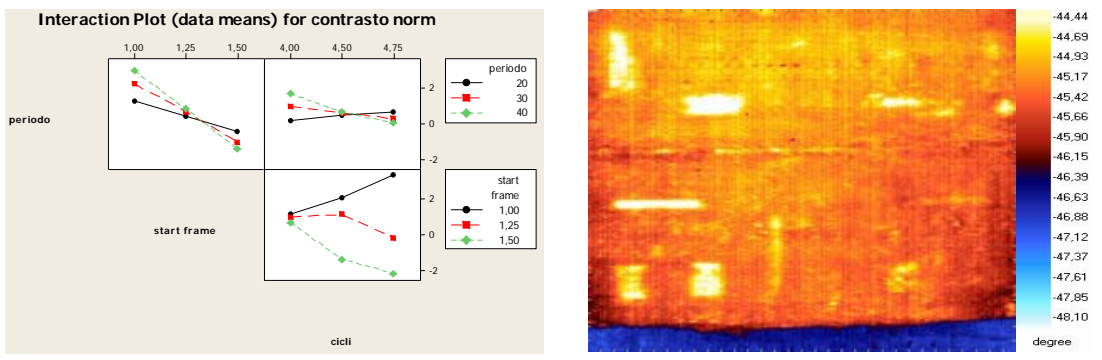

Fig. 11 Normalized contrast interaction plot
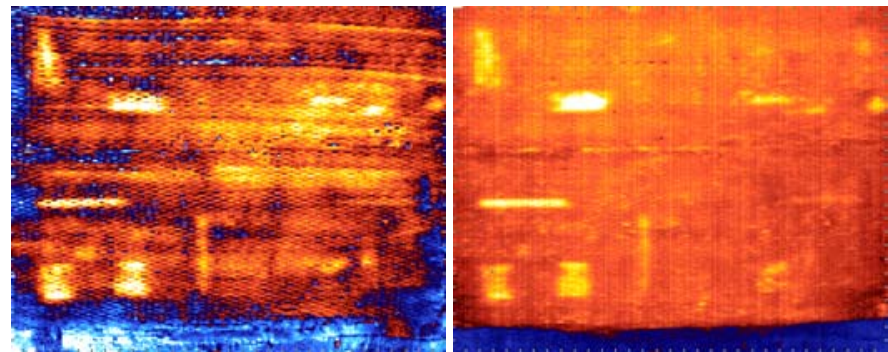

Fig. 12 Phase image for 2 (left) and 2.75 (right) sampled cycles

\subsection{Experimental results}

\subsubsection{Heating period evaluation}

In order to evaluate the heating time that allows the best visibility for different types of defects (delaminations 3, 4 and 8; lack-of-bonding 11, 12 and 15) two parameters were fixed, according to ANOVA results, in the post-processing analysis: 
- $\quad$ start frame corresponding to heating start,

- 5,75 sampled cycles.

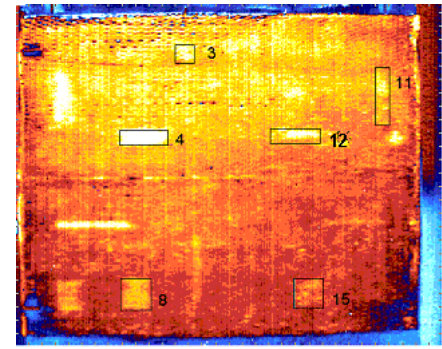

Fig. 13 Defects under analysis

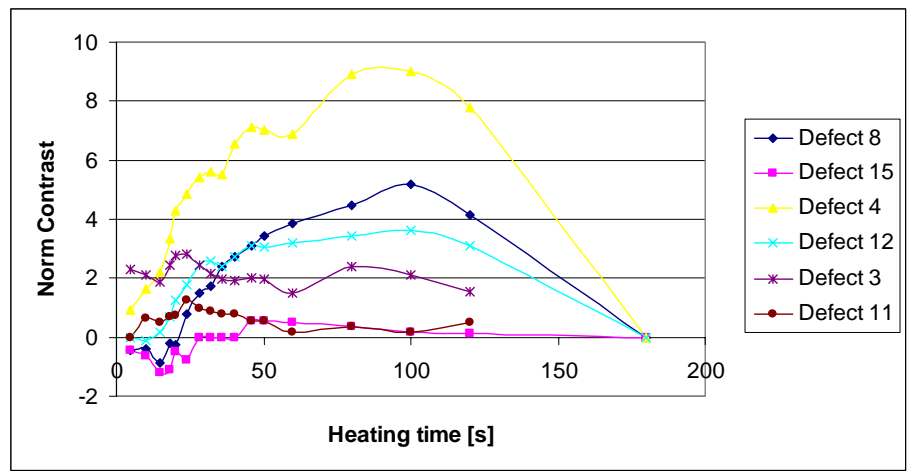

Fig. 14 Normalized contrast variation with heating time

Fig. 14 shows the normalized contrast variation with heating time. It can be pointed out that the normalized contrast gets its maximum value with a heating time of $100 \mathrm{~s}$ for defects 8,4 and 12, of $24 \mathrm{~s}$ for defects 3 and 11 and of $50 \mathrm{~s}$ for defect 15; moreover, if heating time exceeds $180 \mathrm{~s}$ no defect can be detected as the investigated depth is over the FRP-concrete interface. In particular, higher values are obtained for defects 4 and 8 (delamination) characterized by higher thermal resistance.

\subsubsection{Defect dimensions evaluation}

In order to evaluate the shape and dimensions of various types of defects, an upper limit $\left(\phi_{\text {lim }}\right)$ for the phase values was calculated. A pixel must be considered part of a damaged zone if it is characterized by a phase value equal or greater than $\phi_{\text {lim }}$. In particular, two kinds of $\phi_{\text {lim }}$ were calculated, depending on the defect type:

- for delaminations, characterized by higher contrast value, $\phi_{\text {lim }}$ was defined as $\phi_{\lim }=\frac{\bar{\phi}_{d e f}-\bar{\phi}_{\text {sou }}}{2}$, where $\bar{\phi}_{d e f}$ is the average phase value in a defected area; 
- for lack-of-bonding, characterized by lower contrast value, $\phi_{\text {lim }}$ was defined as $\phi_{\lim }=\frac{\frac{\left(\bar{\phi}_{d e f}+\phi_{d e f_{\text {max }}}\right)}{2}-\bar{\phi}_{\text {sou }}}{2}$, where $\phi_{\text {def }_{\max }}$ is the maximum phase value in the defected area and $\bar{\phi}_{\text {sou }}$ is the average phase value for a sound area circumscribing the defect.

\section{$5 \quad$ Lock-in and pulse thermography comparison}

The comparison is referred to those kinds of defects detectable with both techniques. According to Fig. 15 it can be pointed out that lock-in themography can detect delaminations better than pulsed thermography.

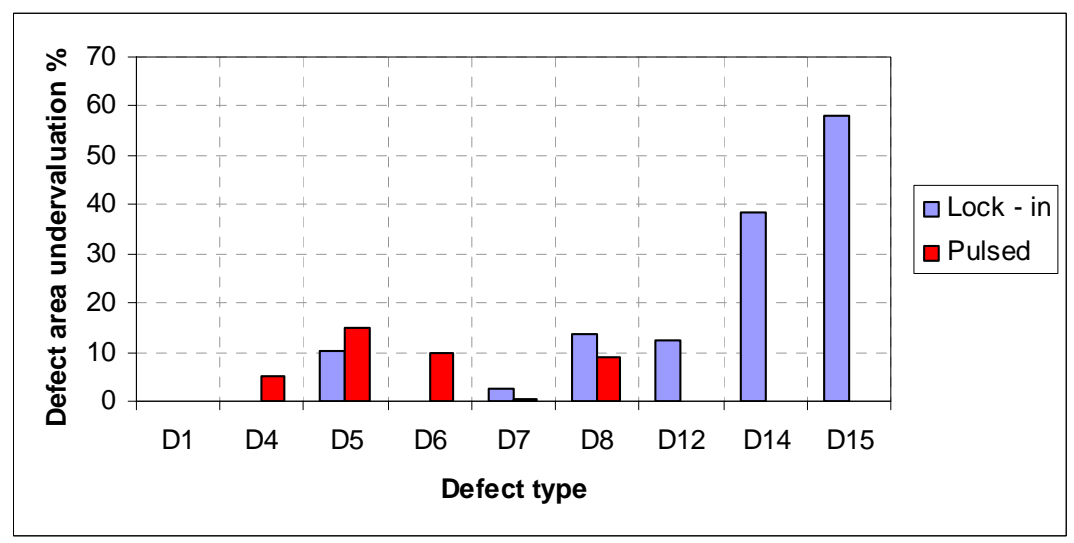

Fig. 15 Lock-in and pulsed thermography comparison

Moreover, unlike pulsed thermography, lock-in thermography is able to estimate lack-of-bonding dimensions, even if these results can't be considered completely reliable.

Finally, lock-in thermography has the great advantage to be quite insensible to the test set-up.

\section{Conclusions}

In this work a reliable and confident non-destructive testing technique, based on infrared thermography, was developed as a tool for the evaluation of hidden defect in FRP-reinforced concrete structures.

A concrete sample strengthened with externally bonded FRP (Fiber Reinforced Plastic) sheets, with known defects appropriately applied at the interface, was analysed both with pulse heating thermography (PT) and lock-in thermography $(\mathrm{LT})$.

According to the experimental results, in order to enhance defect visibility with LT, the following parameters should be adopted: start frame in correspondence to heating start and $(n+0.75)$ numbers of sampled cycles. In fact, it was pointed out, for the first time in literature, that with such parameters, the images are characterized by 
a lower contrast value but, at the same time, also by a lower noise value. This allows the defect to be more visibile.

Moreover, the optimum values of the heating time were pointed out for the different types of defects.

The results obtained by means of infrared thermography, with both procedures, were compared; it seems that LT can detect delaminations better than PT, while the lack-of-bonding dimensions can be estimated only by means of the former technique. Moreover, LT has the great advantage to be quite insensible to the test setup.

Data obtained with PT were post-processed by means of Neural Networks $(\mathrm{NN})$ in order to define the defect shape and dimension; NN don't need interpretation and, once trained, they give results immediately. Nevertheless, NN can give excellent results only after a hard training.

Finally, it can be concluded that the thermographic analysis represents the cheapest, most advantageous and quick technique to be used for in-situ evaluation of the bond quality of FRP-reinforced concrete structure; however the choice of the most appropriate technique, PT or LT, mainly depends on the specific application, with particular regard to the properties of the materials to be investigated.

\section{REFERENCES}

[1] L. De Lorenzis and A. Nanni. Characterization of FRP Rods as Near-Surface Mounted Reinforcement, ASCE Journal of Composites for Construction, 5 (2001) 114-121.

[2] X. Maldague. Theory and Practice of Infrared Technology for Nondestructive Testing. A Wiley-Interscience Publication, John Wiley \& Sons Inc., N.Y. (2001).

[3] D.P. Almond, R. Hamzah, P. Delpech, Peng Wen, M.H. Beheshty and M. B. Saintey. Experimental investigations of defect sizing by transient thermography, QIRT 96-Eurotherm Series 50 ETS ed., Pisa (1997) 233-238.

[4] A. Muscio, S. Marinetti, P.G. Bison, A. Ciliberto, G. Cavacini and E. Grinzato. Modelling of Thermal Non-Destructive Evaluation Techniques for Composite Materials and the European Aerospace Industry. AITA-Advanced Infrared Technology and Applications, Venezia (1999) 143-153.

[5] J.G. Sun. Analysis of quantitative measurement of defects by pulsed thermal imaging. Review of QNDE, D.O. Thompson and D.E. Chimenti Eds., 21 (2002) 572-576.

[6] U. Galietti, V. Luprano, S. Nenna, L. Spagnolo and A. Tundo. Non-destructive defect characterization of concrete structures reinforced by means of FRP, Advanced Infrared Technology and Applications (AITA), Rome (2005).

[7] ICBO. Acceptance criteria for inspection and verification of concrete and reinforced and unreinforced masonry strengthening using fiber-reinforced polymer (FRP) composite systems, ICBO Evaluation Service, INC (2001).

[8] Peters S.T. Handbook of Composites. Chapman \& Hall, London (1998).

[9] M. B. Sainteya and D. P. Almond. An artificial neural network interpreter for transient thermography image data. NDT\&E International, 30 (1997) 291-295.

[10] X. Maldague, Y. Largouet and J.P. Coutier. A study of defect depth using neural networks in pulsed phase thermography: modelling, noise, experiments. Rev. Gén. Therm., 37 (1998) 704-717.

[11] A. Darabi and X. Maldague. Neural network based defect detection and depth estimation in TNDE. NDT\&E International, 33 (2002) 165-175. 